\title{
Wire Rope Reliability Assessment based on Damage Mechanics
}

\author{
Achraf Wahid ${ }^{\mathrm{a}, \mathrm{c}, *}$, Nadia Mouhib ${ }^{\mathrm{a}, \mathrm{b}}$, Abderrazak Ouardi ${ }^{\mathrm{a}}$, Abdelkarim Kartounic, \\ Hamid Chakir ${ }^{\mathrm{c}}$, and Mohamed ELghorba ${ }^{\mathrm{a}}$ \\ ${ }^{a}$ Laboratory of Control and Mechanical Characterization of Materials and Structures, National Higher School of Electricity and Mechanics \\ BP 8118 Oasis, Hassan II University, Casablanca, 20000, Morocco \\ ${ }^{b}$ Institut Suprieur des Etudes Maritimes (ISEM), Km 7 Route d'El Jadida, Casablanca, 20000, Morocco \\ ${ }^{c}$ Condensed Matter Physics Laboratory, Faculty of Sciences Ben M'Sik, University Hassan II of Casablanca, B.P. 7955, Casablanca, 20000, Morocco
}

\begin{abstract}
In order to estimate the instantaneous reliability during a periodic inspection of a lifting wire rope, analytical methods based on experimental results have been developed. Our approach is to study the influence of number of broken strands in the outer layer on the mechanical behavior of the wire rope. To do this, we have evaluated the damage of the wire rope through a static damage model used to predict the lifetime of the steel wire rope. Then, we estimate the reliability by the Weibull law. Finally, we linked the two parameters (damage and reliability) across the life fraction to find the correspondent reliability at each stage of the damage to conduct a predictive maintenance and change the wire rope at the right time.
\end{abstract}

Keywords: non-rotating wire rope; fracture energy; reliability; life fraction; damage; static test

(Submitted on May 20, 2019; Revised on June 22, 2019; Accepted on June 30, 2019)

(C) 2019 Totem Publisher, Inc. All rights reserved.

\section{Introduction}

In service, the failure of a large part of the hoisting wire rope is most often due to the cumulative damage of the wires and strands [1], since the complex stresses can generate local deformations and degradation [2], which can lead to the reduction of the strength of the wire rope as function of time [3], and sometimes leading to a partial or even total fracture.

Several researchers have studied the damage of wire ropes caused by the artificial breaking of wires. Wahid et al. [4] artificially damaged a central core taken from a wire rope in order to analyze the influence of the number of broken wires on its energy-to-break by using the fracture energy corresponding to each percentage of the number of wires broken artificially in the strand. Indeed, they have modeled the damage by Miner, unified theory and static damages. As a result, authors determined three stages of damage, which allows them to predict the moment of acceleration of damage where a predictive maintenance is required.

Mouradi et al. [5] interpreted the residual strength of the non-rotating wire rope in a quantitative sense, using an analytical model to estimate the reliability of the wire rope in terms of its strands cumulative damage. Meksem et al. [6] used a mathematical model based on a probabilistic approach to calculate the reliability of a wire rope from the reliability of its components.

Tijani et al. [7] proposed a new method to study the combined effect of corrosion and fatigue on wire ropes. At the strand scale, they defined the damage evolution as a function of the cumulative deterioration of the wire. To do this, they studied accelerated corrosion by immersion in sulfuric acid for different lengths of time, and estimated the damage from tensile tests on mechanically damaged strands by broken wires.

The purpose of this work is to find a critical life fraction that corresponds to the beginning of the stage of the sudden

\footnotetext{
* Corresponding author.

E-mail address: achraf.wahid90@gmail.com
} 
rupture, which requires predictive maintenance to change the wire rope at the appropriate time, to evaluate the reliability by Weibull's law and to develop a new model of reliability analysis, based on a new relationship between the damage of the rope and its reliability through the life fraction.

\section{Material and Experimental Methods}

\subsection{Material}

In this study, the tests are carried out on a wire rope $19 \times 7$ non-rotation type construction. The first number refers to the number of strands in the rope and the second number refers to the number of wires per strand. The so-called wire rope is made of stainless steel with a metal core [8]; it is used in lifting and many other applications due to its excellent resistance to deformation (Figure 1).

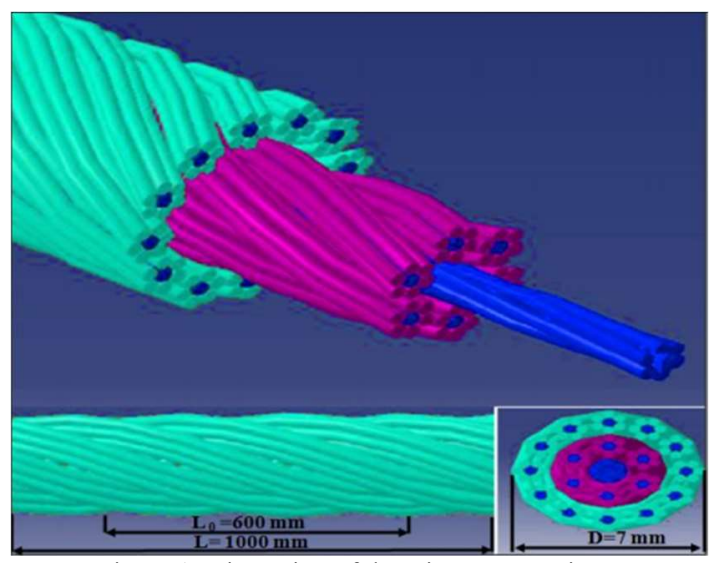

Figure 1. Dimension of the wire rope specimen

\subsection{Experimental Methods.}

The static tensile tests were realized on virgin wire rope specimens and on others artificially damaged by cutting some strand of wire rope from the outer layer until the fracture of the whole outer layer. The minimum length of the specimens used is $600 \mathrm{~mm}$ according to ISO 3108. Therefore, the machine used MTS 64.106 with a capacity of $1000 \mathrm{KN}$ (Figure 2). So, the specimens were tested with imposed displacement corresponding to a strain rate of $2 \mathrm{~mm} / \mathrm{min}$ under the conditions of air and ambient temperature $\left(\approx 20\right.$ to $\left.24^{\circ} \mathrm{C}\right)$.

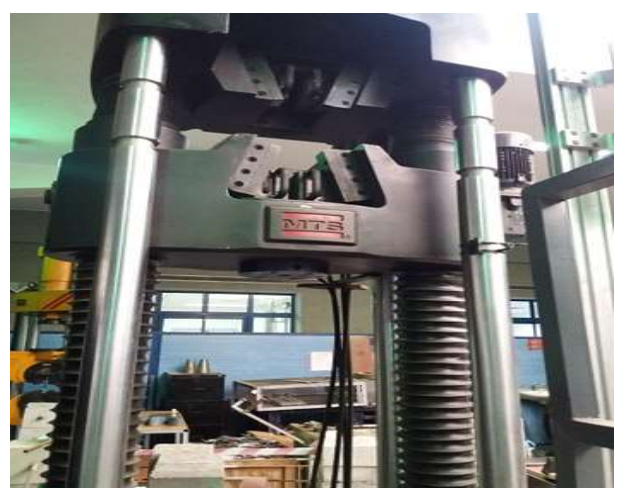

Figure 2. Overview of the MTS 64.106 tensile testing Machine

\section{Results and Discussion}

\subsection{Mechanical Characterizations of the Wire Rope}

The tensile tests performed on the wire rope specimens made it possible to plot the tensile curve, which represents the evolution of the force $(\mathrm{N})$ applied as a function of the displacement $(\mathrm{mm})$. The mechanical characteristics of virgin wire rope are grouped in Table 1. 
Table 1. The mechanical properties of wire rope

\begin{tabular}{|c|c|c|c|c|}
\hline Mechanical properties & Young modulus $(\mathrm{GPa})$ & Elastic limit (MPa) & Tensile strength (MPa) & Strain (\%) \\
\hline Values & 156 & 1470 & 1599 & 1.38 \\
\hline
\end{tabular}

\subsection{Characterization of the Mechanical Behavior of Pre-Damaged Wire Rope}

After the mechanical characterization of the wire rope, a series of tensile tests were carried out on artificially damaged specimens at different levels, i.e., The static tensile were performed on eleven wire rope until 11 artificially cut strands were formed. The curves in Figure 3 describe in 3D the evolution of the force $(\mathrm{kN})$ as a function of displacement $(\mathrm{mm})$ for different levels of damage (number of broken strands).

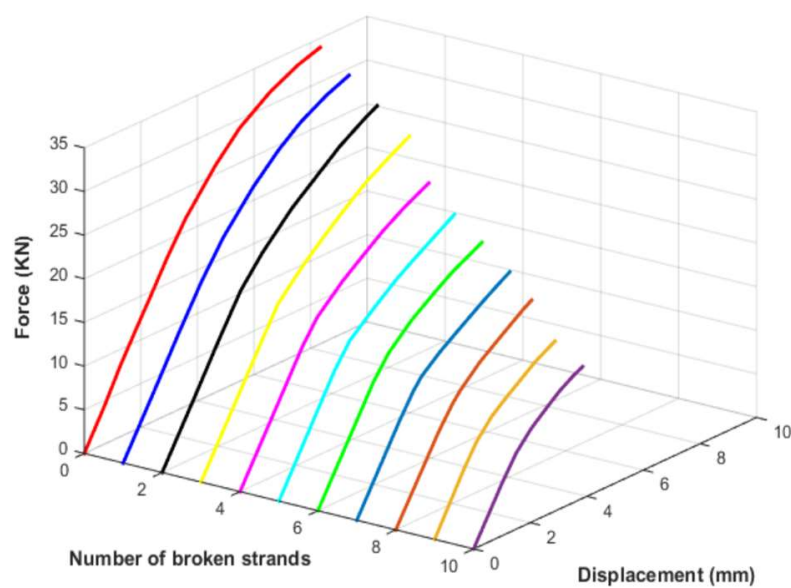

Figure 3. Evolution of the Force $(\mathrm{N})$ versus displacement $(\mathrm{mm})$ for different levels of damage

Figure 3 shows that the residual ultimate force of the wire rope decreases with the increase of the broken strands number in the outer layer; this decrease is accompanied by a decrease in the fracture energy of the wire rope.

\subsection{Lose of Fracture Energy}

To calculate fracture energy, we used the trapezoidal numerical integration method [9] for discrete functions with unequal segments; this method consists of calculating the area under each tensile curve corresponds to each number of broken strands.

The reduction of fracture energy allows us to approach a function which represents the loss of energy according to the life fraction as follows:

$$
U_{u}=a_{1}+a_{2} e^{a_{3} \beta}
$$

$\beta(\%)$ is defined as follows:

$$
\beta=\frac{n_{s}}{N_{s}}
$$

with

- $n_{s}$ : number of broken strands

- $N_{s}$ : number of outer layer strands

We want to estimate the three unknown parameters of the equation: $a_{1,} a_{2}$ and $a_{3}$. The problem is intrinsically nonlinear since we want to minimize.

The residual at each data point $\beta_{i}$ is:

$$
E_{i}=U_{u r i}-\left(a_{1}+a_{2} e^{-a_{3} \beta}\right)
$$


The sum of the square of the residuals is:

$$
f\left(a_{1}, a_{1}, a_{2}\right)=\sum_{i=1}^{n} E_{i}^{2}=\sum_{i=1}^{n}\left(U_{u r i}-\left(a_{1}+a_{2} e^{-a_{3} \beta}\right)\right)^{2}
$$

We assume $a_{3} \neq 0$ is fixed and we put $x_{i}=e^{\left(-a_{3} \beta\right)}$. We use the classical least squares for the linear model and find:

$$
\begin{gathered}
a_{2}=a_{2}\left(a_{3}\right)=\frac{n \sum_{i=1}^{n} U_{u r i} x_{i}-\left(\sum_{i=1}^{n} U_{u r i}\right)\left(\sum_{i=1}^{n} x_{i}\right)}{n \sum_{i=1}^{n} x_{i}^{2}-\left(\sum_{i=1}^{n} x_{i}\right)^{2}} \\
a_{1}=a_{1}\left(a_{3}\right)=\frac{\sum_{i=1}^{n} U_{u r i} \sum_{i=1}^{n} x_{i}^{2}-\left(\sum_{i=1}^{n} U_{u r i} x_{i}\right)\left(\sum_{i=1}^{n} x_{i}\right)}{n \sum_{i=1}^{n} x_{i}^{2}-\left(\sum_{i=1}^{n} x_{i}\right)^{2}}
\end{gathered}
$$

Then we set:

$$
S\left(a_{3}\right)=f\left(a_{1}\left(a_{3}\right), a_{2}\left(a_{3}\right), a_{3}\right)
$$

which means:

$$
S\left(a_{3}\right)=\sum_{i=1}^{n}\left(U_{u r i}-\left(\frac{\sum_{i=1}^{n} U_{u r i} \sum_{i=1}^{n} x_{i}^{2}-\left(\sum_{i=1}^{n} U_{u r i} x_{i}\right)\left(\sum_{i=1}^{n} x_{i}\right)}{n \sum_{i=1}^{n} x_{i}^{2}-\left(\sum_{i=1}^{n} x_{i}\right)^{2}}+\frac{n \sum_{i=1}^{n} U_{u r i} x_{i}-\left(\sum_{i=1}^{n} U_{u r i}\right)\left(\sum_{i=1}^{n} x_{i}\right)}{n \sum_{i=1}^{n} x_{i}^{2}-\left(\sum_{i=1}^{n} x_{i}\right)^{2}} x_{i}\right)\right)^{2}
$$

The optimization problem is now reduced to find the minimum of $S\left(a_{3}\right)$. This can be done using Newton method [10], which makes $a_{3}=-0.8420$. By substituting, we find that $a_{1}=-110.2160$ and $a_{2}=290.0767$.

Equation (1) becomes:

$$
U_{u r}=-110.2160+290.0767 e^{(-0.8420 \beta)}
$$

The obtained equation makes it possible to follow the loss of fracture energy at each life fraction, as seen in Figure 4.

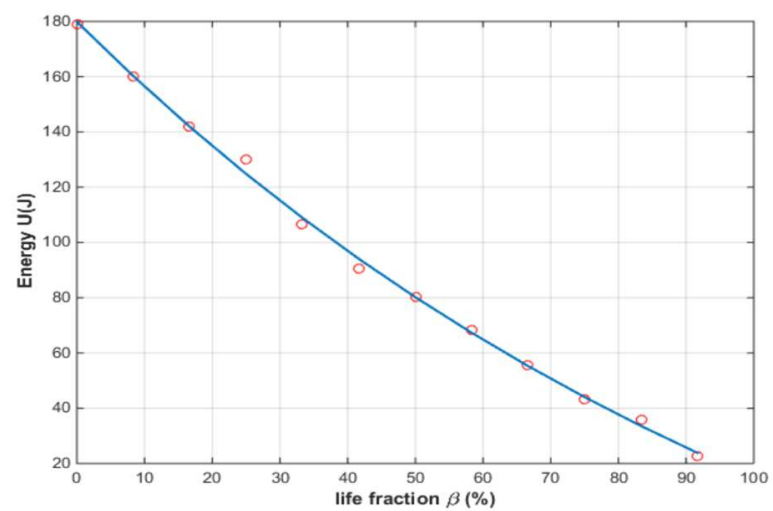

Figure 4. Energy loss as a function of life fraction

We notice that there is a very remarkable decrease in the energy of rupture with the evolution of the artificial damage represented by the life fraction. It is clear that the behavior of the wire rope is impacted by the presence of the artificial 
damage of the strands, which lead to the degradation of mechanical properties of the wire rope and consequently tends to become more brittle which leads to an increasingly brutal rupture.

\subsection{Damage}

To assess the degree of the wire rope's degradation, it is necessary to measure the level of damage. This last one is directly related to the physical properties; hence, the importance of adopting a model of the damage depends on the state of stress of the studied structure, which is linked to the life fraction $\beta$. Therefore, the choice of the model of static damage whose expression is as follows [11]:

$$
D_{s}=\frac{1-\frac{U_{u r}}{U_{u}}}{1-\frac{U_{a}}{U_{u}}}
$$

- $U_{u r}$ : residual ultimate fracture energy

- $U_{u}$ : ultimate fracture energy of virgin material

- $U_{a}$ : fracture energy corresponds to the breaking of the last strand of the layer

The variation of the static damage as a function of the percentages of broken strands in the outer layer of the test samples (corresponding to 1 to 12 strands constituting the outer layer) is shown in Figure 5.

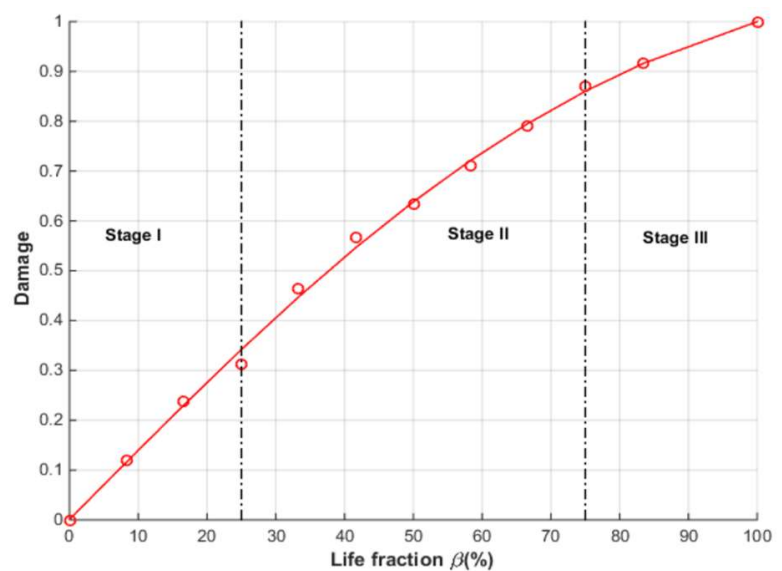

Figure 5. Evolution of the static damage as a function of life fraction

From the curve above, we can see that the damage is an increasing function as a function of the life fraction; it is higher for greater values of the life fraction until the total failure of the outer layer. The change of curvature of the damage evolution allowed us to distinguish three stages of damage. The first stage corresponds to the initiation of the damage for a life fraction between 0 and 28\%; in this stage the damage grows slowly. The second stage is in the range of $\beta=[28 \%$, 75\%] where the damage becomes progressive and predictive maintenance is required in the industrial. The life fraction reaches a critical value corresponds to the end of this stage and the beginning of the third stage where the damage accelerates and the failure can be brutal.

\subsection{Reliability}

To find a formula that links damage and reliability across the life fraction, we must write the two parameters according to the life fraction. We start with the expression of reliability which is an explicit function of time according to Weibull's law [12]:

$$
R=\exp \left(-\left(\frac{t-\gamma}{\eta}\right)^{\lambda}\right)
$$

The simplified expression of the reliability in term of the life fraction, it is given by the following relationship [5-6, 12]: 


$$
R(\beta)=\exp \left(-\beta^{\lambda}\right)
$$

Figure 6 illustrates the superposition of the damage and reliability curves as a function of the life fraction.

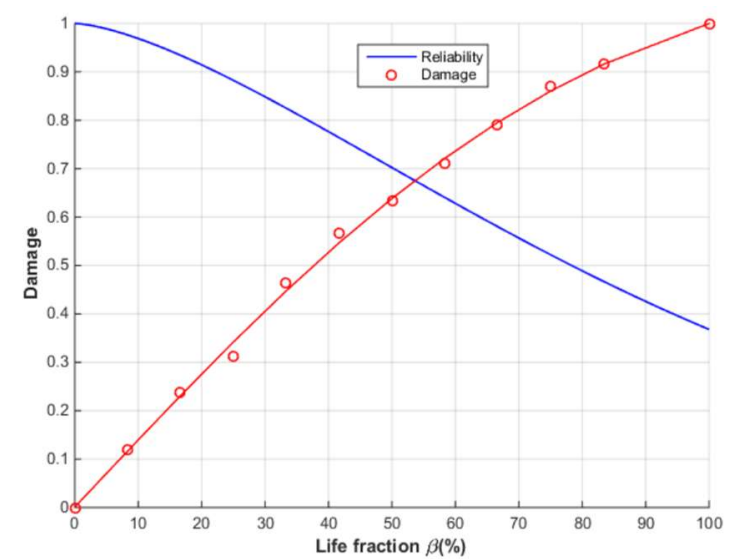

Figure 6. Reliability and damage curves as a function of life fraction

Figure 6 shows that the reliability varies in the opposite direction of the damage, which means that there is a relationship that must be determined between the two parameters. For a fraction of life less than about $53 \%$, we note that the damage increases but remains below the reliability; this trend is reversed beyond this life fraction. In addition, we can see the intersection point of the two curves representing damage and reliability is in Stage II. For a life fraction $\beta=1$, the reliability is not equal zero. This corresponds to a residual reliability just before the material fracture.

\subsection{Reliability-Damage Relationship}

To express the damage as a function of the life fraction, we rewrite the Equation (3) in the following form:

$$
D=\frac{U_{u}-U_{u r}}{U_{u}-U_{a}}
$$

then

$$
\begin{aligned}
& D\left(U_{u}-U_{a}\right)=U_{a}-U_{u r} \\
& U_{u}-D\left(U_{u}-U_{a}\right)=U_{u r}
\end{aligned}
$$

We replace $u r$ by its expression in Equation (3) and get:

$$
\begin{gathered}
a_{1}+a_{2} e^{a_{3} \beta}=U_{u}-D\left(U_{u}-U_{a}\right) \\
e^{a_{3} \beta}=\frac{1}{a_{3}}\left(U_{u}-D\left(U_{a}-U_{u}\right)-a_{1}\right)
\end{gathered}
$$

After simplification:

$$
\beta=\frac{1}{a_{3}} \log \left(\frac{1}{a_{2}}\left(U_{u}-D\left(U_{a}-U_{u}\right)-a_{1}\right)\right)
$$

We finally get the expression of reliability as a function of the damage:

$$
R=e^{\left(\frac{1}{a_{3}} \log \left(\frac{1}{a 2}\left(U_{u}-D\left(U_{a}-U_{u}\right)-a_{1}\right)\right)\right)^{2}}
$$


From Equation (8), we plot the reliability curve as a function of damage:

Figure 7 shows that the reliability decreases with the progressive increase of the failure criterion. On the other hand, we note that when the damage gets its maximum value, which is equal to 1 , the reliability is (1/e). This means that the damage theory considers that the damage reaches the value 1 when there is a critical degradation but the material can keep a certain quantity of absorbing energy explained by a non-zero reliability; the latter becomes zero when the material is completely destroyed.

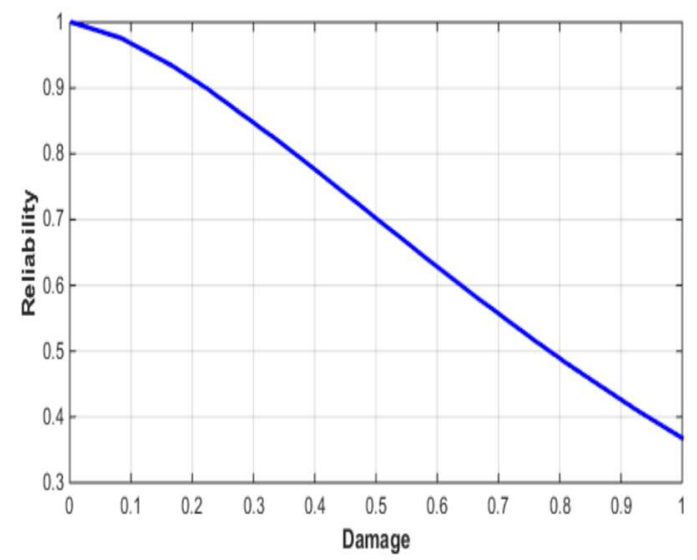

Figure 7. Graphical representation of reliability as a function of damage

\section{Conclusions}

In this article, we followed the evolution of wire rope damage at different levels of its outer layer degradation. The damage curve helped us determine three stages of damage and define the critical life fraction $\beta \mathrm{c}$ which corresponds to the acceleration of the damage. We estimated the reliability of the wire rope based on the Weibull law. In addition, we reported a reliability analysis of the wire rope via a new approach based on a nonlinear regression of the wire rope's energy loss evolution in order to express the life fraction according to the damage. As a result, reliability can be expressed as a function of the damage. This makes it possible to estimate reliability at each value of the damage. It can also predict the damage for a given reliability.

\section{References}

1. C. Asmâa, E. L. G. Mohamed, B. Abdelkader, and H. Abdelilah, "Estimated Loss of Residual Strength of a Flexible Metal Lifting Wire Rope: Case of Artificial Damage," Recent Advances in Mechanical Engineering and Mechanics, pp. 160, 2014

2. X. D. Chang, Y. X. Peng, Z, C. Zhu, S. Y. Zou, X. S. Gong, and C. M. Xu, "Effect of Wear Scar Characteristics on the Bearing Capacity and Fracture Failure Behavior of Winding Hoist Wire Rope," Tribology International, Vol. 130, pp. 270-283, 2019

3. A. Vorontsov, V. Volokhovsky, et al., "Prediction of Operating Time of Steel Wire Ropes using Magnetic NDT Data," in Proceedings of OIPEEC Conference, Johannesburg, 2007

4. A. Wahid, N. Mouhib, A. Kartouni, and H. Chakir, "Energy Method for Experimental Life Prediction of Central Core Strand Constituting a Steel Wire Rope," Engineering Failure Analysis, Vol. 97, pp. 61-71, 2018

5. H. Mouradi, A. EI. Barkany, and A. EI. Biyaali, "Reliability Estimation of Non-Rotating Ropes based on Cumulative Damage," International Journal of Performability Engineering, Vol. 12, No. 5, pp. 471-480, 2016

6. A. Meksem, M. EI. Ghorba, A. Benali, and A. EI. Barkany, "Optimization by the Reliability of the Damage by Tiredness of a Wire Rope of Lifting," Applied Mechanics and Materials, Vol. 61, 2011

7. T. Amal, M. Meknassi, H. Chaffoui, and M. Elghorba, "Combined Effect of Broken Rope Components and Corrosion on Damage Evolution Through Its Lifetime,” Journal of Materials in Civil Engineering, Vol. 29, No. 7, pp. 04017035, 2017

8. A. K. Kaw, E. K. Kalu, and D. Nguyen, "Numerical Methods with Applications: Abridged," University of South Florida, 2009

9. S. J. Ahn, "Least Squares Orthogonal Distance Fitting of Curves and Surfaces in Space," Springer Science \& Business Media, Vol. 3151, 2004

10. N. Mouhib, H. Ouaomar, M. Lahlou1, and M. El Ghorba, "Characterization of Residual Energy Loss and Damage Prediction of 7-Wire Strand Extracted from a Steel Wire Rope and Subjected to a Static Test," International Journal of Research, Vol. 2, No. 6, pp. 473-478, 2015

11. J. McCool, "Using the Weibull Distribution: Reliability, Modeling, and Inference," John Wiley \& Sons, Vol. 950,2012

12. M. Chahid, et al., "Optimisation Fiabiliste De L'endommagement En Fatigue D'un Matériau Composite Fibres De Verreépoxyde-Cas Des Systèmes Séries Et Parallèles,” Matériaux \& Techniques, Vol. 84, No. 11-12, pp. 13-17, 1996 